\title{
Frequency-dependent line model in the time domain for simulation of fast and impulsive transients
}

\author{
Pablo Torrez Caballero ${ }^{a}$, Eduardo C. Marques Costa ${ }^{\mathrm{b}, *}$, Sérgio Kurokawa ${ }^{\mathrm{a}}$ \\ ${ }^{a}$ Unesp - Univ. Estadual Paulista, Faculdade de Engenharia de Ilha Solteira - FEIS, Departamento de Engenharia Elétrica, Ilha Solteira, SP, Brazil \\ ${ }^{\mathrm{b}}$ Universidade de São Paulo - USP, Escola Politécnica, Departamento de Engenharia de Energia e Automação Elétricas - PEA, São Paulo, SP, Brazil
}

\section{A R T I C L E I N F O}

\section{Article history:}

Received 19 May 2014

Received in revised form 3 December 2015

Accepted 2 January 2016

Available online 11 February 2016

\section{Keywords:}

Bergeron method

Transmission line modeling - TLM

Electromagnetic transients

Time-domain analysis

\begin{abstract}
A B S T R A C T
A new transmission line model is proposed based on the well-established Bergeron method. The conventional Bergeron model is characterized by the line representation by concentrated longitudinal and transversal parameters, i.e., electrical parameters of the line are represented by means of electric circuit elements. The original approach of this research is the inclusion the frequency effect in the longitudinal parameters of the Bergeron line representation. In order to increase the frequency range covered by the proposed model, the line is represented by a cascade of line segments which are modeled following the proposed frequency-dependent Bergeron circuit. The differential equations resulted from the proposed development are represented by state matrices. The line representation by cascade of frequency-dependent Bergeron circuits enables to extend the application of the new modeling technique for simulations considering a wide range of frequencies, from a switching up to an atmospheric impulse. The proposed line model is validated based on results obtained from the well-established line model using numerical Laplace transform.
\end{abstract}

(c) 2016 Elsevier Ltd. All rights reserved.

\section{Introduction}

There are several transmission line models available in the technical literature to study electromagnetic transients in power transmission systems. Basically, these models may be classified into two general groups: by lumped parameters or by distributed parameters.

In the first group, transmission lines are modeled from the representation by lumped elements, i.e., line is modeled by an equivalent representation by means of electric circuits composed of resistive, inductive and capacitive elements. These models are developed directly in the time domain, which means that can be applied for transient simulations including time-variable and non-linear elements, as: metal-oxide surge arresters, relays, nonlinear loads and many other power components. This characteristic is the principal advantage in the transmission line modeling (TLM) by lumped elements [1]. The line representation by lumped parameters is well established in the technical literature for simulation of electromagnetic transients as well as other applications for power flow studies, fault location through long transmission lines and steady state phenomena [2-4].

\footnotetext{
* Corresponding author.

E-mail addresses: educosta@pea.usp.br (E.C. Marques Costa), kurokawa@dee. feis.unesp.br (S. Kurokawa).
}

The line modeling by distributed parameters is developed directly from the frequency-dependent parameters of the line representation by two-port circuit in the frequency domain. From this approach, the line modeling and simulations are carried out in the frequency domain and time-domain results are obtained using numeric transforms [5]. The frequency-dependent parameters of the line are accurately represented using frequency-domain models; however, these models have restrictions for inclusion of time-variable elements in the simulation process, since most power components are well established and modeled in the time domain [6].

Despite line models by lumped elements are developed in the time domain, the frequency effect on the longitudinal parameters can be included in the model using fitting methods. New frequency-dependent models based on the electric circuit approach have been described in the technical literature on TLM. These models are developed directly in the time domain from the line representation by cascade of $\pi$ circuits, where the frequency effect on the electrical parameters is fitted by rational functions $R_{f i t}(\omega)$ and $L_{f i t}(\omega)$ (resistance and inductance) based on the longitudinal impedance of the line $Z(\omega)$, which is calculated taken into account the earth-return impedance (soil effect) and the skin effect on the cables. For example, the frequency-dependent line model described in Refs. [7,8] shows to be robust and accurate for most of transient conditions on power transmission systems. 
However, depending of the transmission system characteristics (source, line and load) and transient conditions, the frequencydependent model based on cascade of lumped elements shows to be costly in computational terms, depending of the quantity of line equivalent circuits in the cascade, total simulation time and integration step. Furthermore, hard unbalanced conditions could lead to some inaccuracies because the multi-phase modeling using a constant and real transformation matrix for the line decoupling into the respective propagation modes. Thus, the line representation by frequency-dependent cascade of $\pi$ circuits shows to be efficient for several situations; however, some restrictions in the modeling procedure and inaccuracies are observed for specific cases. From this last statement, the same fitting procedure in Refs. $[7,8]$ is also applied for the proposed line model based on the Bergeron method for simulation of fast and impulsive transients.

The Bergeron method, also known as method of the characteristics, was firstly proposed to solve hydraulic systems and after applied to electrical problems, more specifically, electromagnetic wave propagation along a lossless line [9]. In this case, the line modeling is carried out considering only the longitudinal inductance $L$ and the shunt capacitance $C$, which means that the line resistance $R$ and transversal conductance $G$ are neglected. Thereafter, H.W. Dommel proposed a nodal solution combining the method of the characteristics for transmission lines with losses and the integration method of the trapezoidal rule for lumped parameters. The losses in the Bergeron's line model were represented by constant lumped resistances located at the sending and receiving ends of the equivalent circuit. The Bergeron's method with losses was included in the well-known Electromagnetic Transient Program (EMTP) [10].

A first approach for inclusion of the frequency effect in transmission line models, direct in the time domain, was described in [11]. An extension of the Bergeron's method of characteristics was developed for transmission lines with frequency-dependent parameters. However, the frequency-dependent parameters were included in the line model using inverse transforms and convolutions, which also results in several restrictions in simulations of time-variable power components and non-linear phenomena.

The proposed model is represented by a cascade of Bergeron's circuits, which results in an accurate line model capable of simulating electromagnetic transients composed of a wide range of frequencies, differently of most models developed by lumped parameters and the classical Bergeron model itself. Thus, the inclusion of the frequency effect in the Bergeron model and the line representation by cascade of lumped elements are the main contribution of this research.

This paper is structured into three parts. The first part is an introduction of the classical Bergeron model for transmission lines without losses and for lossy lines using constant parameters. The second part describes the inclusion of the frequency effect in the Bergeron model using vector fitting and the line representation by cascade of frequency-dependent Bergeron circuits. The third part validates the proposed time-domain model comparing results with a well-established model using numerical Laplace transform (NLT line model) [5]. Two signals are evaluated for the two line models: switching impulse (composed of low frequencies) and an atmospheric impulse (composed of low up to very high frequencies).

\section{The Bergeron line model}

The Bergeron's method was firstly applied for lossless transmission lines. This means that only the line inductance per unit of length (p.u.l.) $L^{\prime}$ and the p.u.l. capacitance $C^{\prime}$ were included in the model, whereas the longitudinal p.u.l. resistance $R^{\prime}$ and the p.u.l. conductance $G^{\prime}$ were neglected. In fact, the method of the characteristics could be applied for lossy transmission lines, but the resulting ordinary differential equations could not be directly integrated. Thus, considering a single-phase line with length $l$, the current and voltage at a point $x$ along the line are expressed as follows:

$$
\begin{gathered}
-\frac{\partial e}{\partial x}=L^{\prime} \frac{\partial i}{\partial t} \\
-\frac{\partial i}{\partial x}=C^{\prime} \frac{\partial e}{\partial t}
\end{gathered}
$$

The first hand of (2) and (3) represents the voltage and the current as a function of the distance $x$ along the line, i.e., voltage and current wave propagation along the line as a function of the time $t$.

The general solutions of (1) and (2) are expressed [9]:

$i(x, t)=f_{1}(x-v t)+f_{2}(x+v t)$

$e(x, t)=Z_{0} f_{1}(x-v t)+Z_{0} f_{2}(x+v t)$

Terms $f_{1}$ and $f_{2}$ are arbitrary functions of $(x \pm v t)$. Function $f_{1}$ represents the forward wave propagation along the line with velocity $v$ (also known as propagation or phase velocity) whereas $f_{2}$ represents the wave propagation in a back forward direction. The line characteristic impedance $Z_{0}$ and the propagation velocity $v$ are expressed in the technical literature as [10]:

$Z_{0}=\sqrt{\frac{L^{\prime}}{C^{\prime}}} ; \quad v=\frac{1}{\sqrt{L^{\prime} C^{\prime}}}$

Multiplying (3) by the characteristic impedance $Z_{0}$ and adding in (4), the following formulation is obtained [9]:

$e(x, t)+Z_{0} i(x, t)=2 Z_{0} f_{1}(x-v t)$

$e(x, t)-Z_{0} i(x, t)=-2 Z_{0} f_{2}(x+v t)$

Analyzing (6), $\left(e+Z_{0} i\right)$ is constant for $(x-v t)$. The same instance is valid for the voltage $\left(e-Z_{0} i\right)$ in association with $(x+v t)$. These constants are intrinsic related to the propagation characteristics and differential equations of a lossless transmission line.

Since $(x \pm v t)$ is constant, the traveling time of an electromagnetic wave from the sending end to the receiving end of the line is also constant and is expressed as:

$\tau=\frac{l}{v}=l \sqrt{L^{\prime} C^{\prime}}$

The equivalent circuit for a lossless line is described in Fig. 1.

The forward wave is constant from the node $k$ to the node $m$ at instant $t-\tau$. Analogously for a back forward wave from the node $m$ to $k$. This means that the forward wave, which takes $\tau$ seconds to travel from the sending end $k$ to the receiving end $m$, has the same magnitude than the back forward wave from terminal $m$ to $k$ because no losses are considered in the line modeling which leads to an undefined number of wave reflections between the line terminals [9].

The historical currents for the Bergeron's line model without losses, defined in Fig. 1, are expressed as [10]:

$I_{k, m}(t)=\frac{1}{Z_{0}} e_{k}(t)-I_{k}(t-\tau)$

$I_{m, k}(t)=\frac{1}{Z_{0}} e_{m}(t)-I_{m}(t-\tau)$

Terms $I_{k}$ and $I_{m}$ are equivalent current sources at the sending and the receiving ends of the line, respectively. Sources $I_{k}$ and $I_{m}$ 


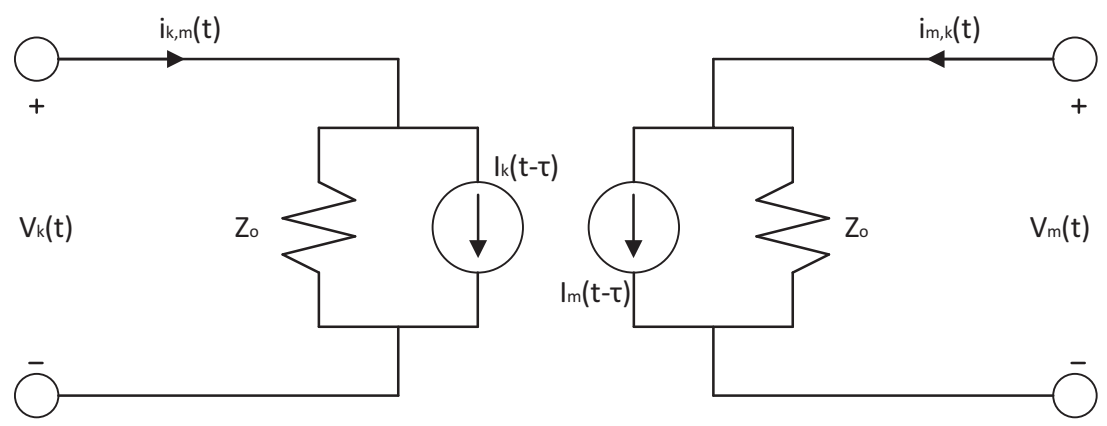

Fig. 1. Equivalent impedance circuit of the Bergeron line model.

are known at state time $t$ from the past history at time $t-\tau$, as expressed in (11) and (12), respectively.

$I_{k}(t-\tau)=-\frac{1}{Z_{0}} e_{m}(t-\tau)-i_{m, k}(t-\tau)$

$I_{m}(t-\tau)=-\frac{1}{Z_{0}} e_{k}(t-\tau)-i_{k, m}(t-\tau)$

Eqs. (9)-(12) are the time-domain formulation for a lossless line represented by the equivalent impedance circuit in Fig. 1. However, the losses can be represented by concentrated lumped resistances at the sending and receiving ends of the line, as shown in Fig. 2.

The total series impedance is concentrated at both terminals of the line represented by the equivalent impedance circuit in Fig. 2. Thus, (11) and (12) can be reformulated [11]:

$I_{k}(t-\tau)=-\frac{1}{\left(Z_{0}+R / 2\right)} e_{m}(t-\tau)-i_{m, k}(t-\tau)$

$I_{m}(t-\tau)=-\frac{1}{\left(Z_{0}+R / 2\right)} e_{k}(t-\tau)-i_{k, m}(t-\tau)$

The term $R / 2$ is added to the characteristic impedance $Z_{0}$. Term $R$ is the total resistance of the line, where part is concentrated at the node $k$ and the other half at the node $m$, as described in Fig. 2. Although this approach has been used in most EMTP versions, entitled as Bergeron model, the concentrated resistance $R$ is constant, which means that the Bergeron model for lossy lines has not properly taken into account the frequency effect on the longitudinal parameters. Most transient phenomena in power sys- tems are composed of a wide range of frequencies which means that the conventional Bergeron model has restrictions in simulations of fast and impulsive transients.

\section{Including the frequency effect in the Bergeron model}

As well-established in the technical literature on TLM, the impedance of transmission lines is highly dependent of the frequency due to the earth-return current (soil effect) and the skin effect in the conductor. This means that the transmission lines are characterized by a longitudinal impedance $Z(\omega)$, resulted from the soil and skin effects $[5,7,8]$. Thus, the inclusion of the frequency effect in transmission line models is an important issue for simulation of electromagnetic transients.

The proposed frequency-dependent Bergeron model is described in details in this section. Initially, a brief review on vector fitting is presented in order to show how the frequencydependent parameters are synthesized for direct representation in the time domain by means of a single rational function and lumped elements. In sequence, the new Bergeron line model is described step by using the proposed fitting method.

\section{Fitting the longitudinal impedance}

The fitting procedure consists in the approximation of a rational function $Z_{f i t}(\omega)$ to the longitudinal impedance of the line $Z(\omega)$. The poles, zeros and residues of the fitted impedance $Z_{\text {fit }}(\omega)$ are associated with a $R L$ circuit, which is the main step to represent the frequency effect in TLM in the time domain.

The referred equivalent circuit is described in Fig. 3.

The rational function that represents the equivalent $R L$ circuit in Fig. 3 is expressed as $[7,8,12]$ :

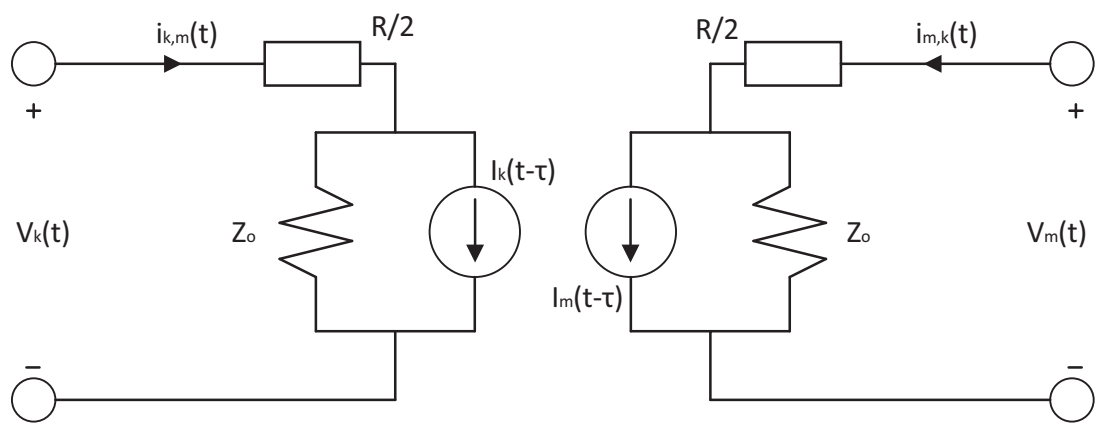

Fig. 2. Equivalent impedance circuit considering the line losses. 


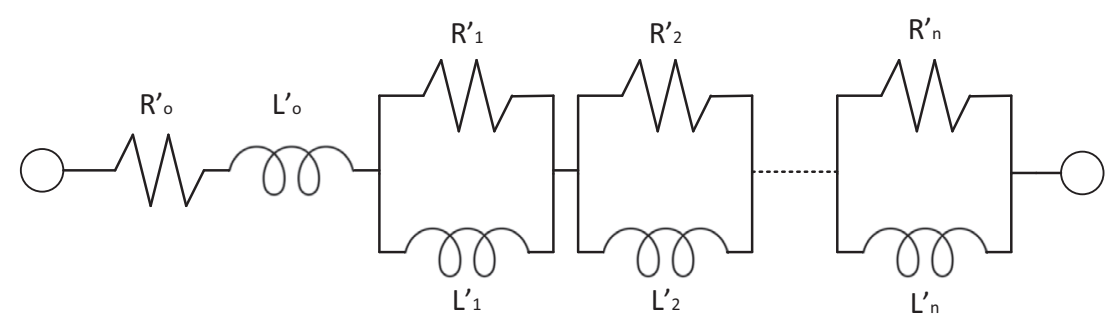

Fig. 3. Equivalent circuit for fitting the frequency-dependent line impedance.

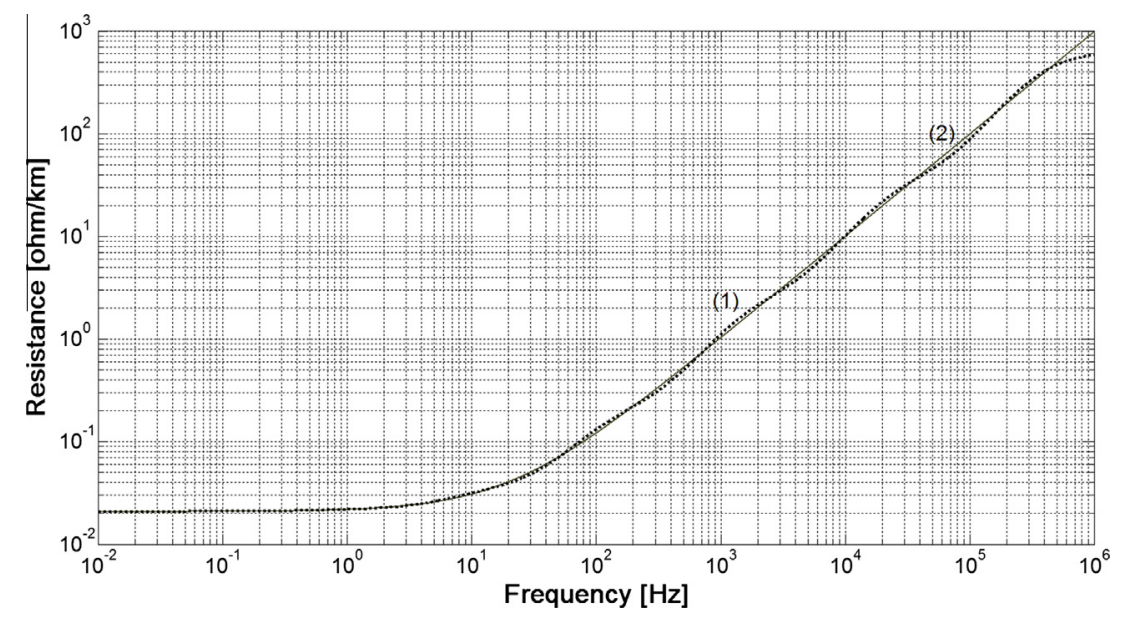

Fig. 4. Line p.u.l. resistance $R(\omega)$ (curve 1) and fitted p.u.l. resistance $R_{f i t}(\omega)$ (curve 2).

$\mathrm{Z}(\omega) \approx \mathrm{Z}_{f i t}(\omega)=R_{0}^{\prime}+j \omega L_{0}^{\prime}+\sum_{i=1}^{n} \frac{j \omega R_{i}^{\prime}}{\left(j \omega+R_{i}^{\prime} / L_{i}^{\prime}\right)}$.

Term $\omega$ is the angular velocity. Terms $R_{0}^{\prime}$ and $L_{0}^{\prime}$ are the p.u.l. resistance and inductance for $\omega=0$, respectively. The frequency range is fitted as a function of the quantity of $R L$ blocks in the electric circuit in Fig. 3.

A single propagation mode is characterized by a Grosbeak conductor at $28 \mathrm{~m}$ above the ground with soil conductivity of $1000 \Omega \mathrm{m}$. Thus, the propagation mode can be considered as a single-phase line. The calculated resistance $R(\omega)$, taken into account the ground and skin effects, and the fitted resistance $R_{f i t}(\omega)$ are obtained for frequencies up to $1 \mathrm{MHz}$, as demonstrated in Fig. 4.

The calculated and fitted inductances $L(\omega)$ and $L_{\text {fit }}(\omega)$, respectively, are in Fig. 5.

The resistance and inductance fitting show to be accurate compared with the same values calculated using the conventional approach for calculation of transmission line parameters [4]. The algorithm applied for calculation of the residues and poles of the rational function $Z_{f i t}(\omega)$ is the well-established Vector Fitting [12]. This algorithm has shown to be accurate and robust for smooth and resonant responses with high order and wide frequency bands. Furthermore, the same method has been efficient in the development of time-domain line models for simulation of electromagnetic transients in power systems $[1,7,12]$.

\section{Frequency-dependent Bergeron line model}

The proposed development consists basically to replace the constant resistance, referred to the line losses in Fig. 2, by the equivalent circuit described in Fig. 3. This procedure requires a new formulation for the Bergeron model which results in a system of differential equations with dimension as long as the number of RL blocks in the equivalent circuit in Fig. 3.
Substituting the resistance $R$ (Fig. 2) by the equivalent circuit expressed by $Z_{\text {fit }}(\omega)$, the frequency-dependent Bergeron circuit is restructured in Fig. 6.

The frequency-dependent impedance is concentrated at the nodes $k$ and $m$ of the equivalent circuit, from the same way described for the Bergeron model with losses. However, the constant resistance $R$ is replaced by the impedance $Z_{f i t}(\omega)$, in (15), directly in the time domain.

The number of $R L$ blocks is defined based on the type of electromagnetic transient to be analyzed. For an input signal composed of low frequencies, e.g. a switching operation, no more than three or four $R L$ circuits are necessary. Otherwise, for fast and impulsive transients, as an atmospheric impulse for example, several $R L$ blocks are required to cover the entire frequency range which a steep-front wave is composed.

Considering the frequency-dependent Bergeron circuit in Fig. 6, the relationship of voltage on the resistors and the inductors in the node $k$ is expressed in (16)-(18).

$\frac{R_{1}}{2}\left(i_{k 0}-i_{k 1}\right)=\frac{L_{1}}{2} \frac{d i_{k 1}}{d t}$

$\frac{R_{2}}{2}\left(i_{k 0}-i_{k 2}\right)=\frac{L_{2}}{2} \frac{d i_{k 2}}{d t}$

$\frac{R_{n}}{2}\left(i_{k 0}-i_{k n}\right)=\frac{L_{n}}{2} \frac{d i_{k n}}{d t}$

In (16)-(18), terms $i_{k 0}, i_{k 1}$ to $i_{k n}$ are the currents through the resistor $R_{0}$ and inductors $L_{1}$ to $L_{n}$, respectively. Following the Kirchoff's current law, the current through the resistors $R_{1}$ and $R_{n}$ are $\left(i_{k 0}-i_{k 1}\right)$ and $\left(i_{k 0}-i_{k n}\right)$, respectively. Thus, the general expression is developed associating the past-history current source $I_{k}(t-\tau)$ to the state current $i_{k 0}$, which is equivalent to the current $i_{k, m}(t)$ in Fig. 6 . 


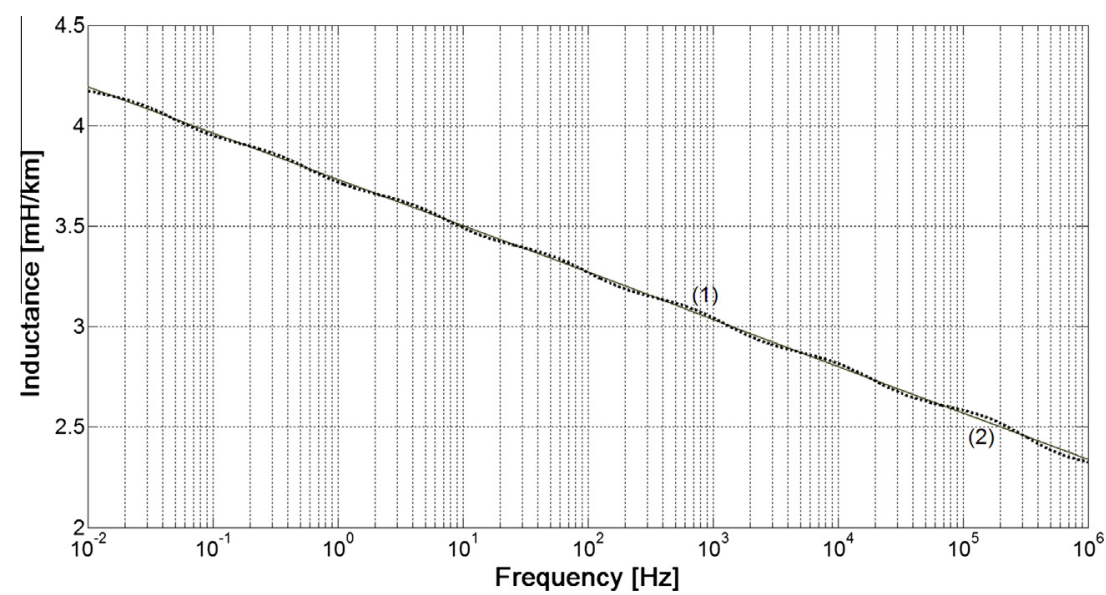

Fig. 5. Line p.u.l. inductance $L(\omega)$ (curve 1) and fitted p.u.l. inductance $L_{\text {fit }}(\omega)$ (curve 2).

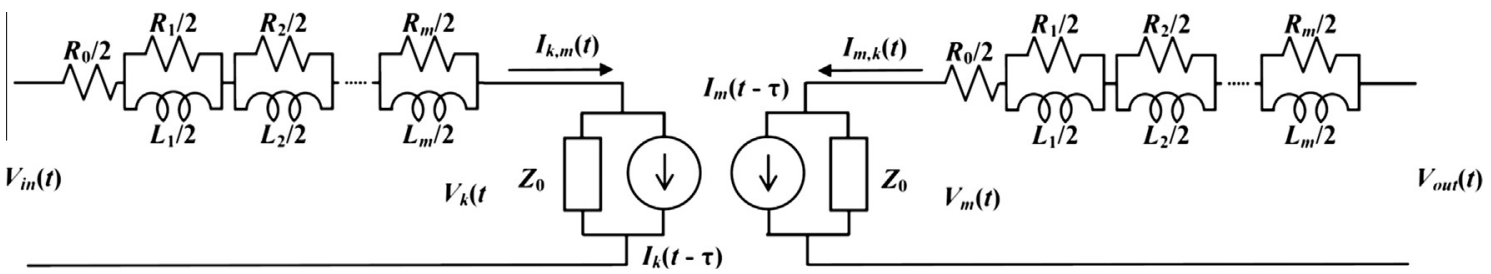

Fig. 6. Frequency-dependent Bergeron circuit.

$$
V_{s}-R_{0} i_{k 0}-R_{1}\left(i_{k 0}-i_{k 1}\right)-\cdots-R_{n}\left(i_{k 0}-i_{k n}\right)+Z_{0} I_{k}(t-\tau)=Z_{0} i_{k 0}
$$

The voltage source $V_{S}$ in (19) represents the input voltage $V_{\text {in }}(t)$ in the frequency-dependent Bergeron's circuit in Fig. 6. The firstorder system, based on (16)-(19), can be presented as state equations from the following form:

$\left[\dot{\mathrm{I}}_{k}\right]=\left[\mathrm{A}_{k}\right]\left[\mathrm{I}_{k}\right]+\left[\mathrm{B}_{k}\right][\mathrm{S}]$

Vector $\left[\mathrm{I}_{k}\right]$ is composed of the currents in inductors $L_{1}$ to $L_{n}$, its transposed form is expressed as:

$\left[\mathrm{I}_{k}\right]^{T}=\left[\begin{array}{llll}i_{k 1} & i_{k 2} & \ldots & i_{k n}\end{array}\right]$

The current derivatives from (21) are expressed by the following transposed vector:

$\left[\dot{\mathrm{I}}_{k}\right]^{T}=\left[\frac{d i_{k 1}}{d t} \frac{d i_{k 2}}{d t} \ldots \frac{d i_{k n}}{d t}\right]$

The state matrix $\left[A_{k}\right]$ is constant and expressed as a function of $R$ and $L$ values of the circuit in Fig. 3 and also from the characteristic impedance $Z_{0}$, calculated based on the line parameters for direct current. Matrix $[\mathrm{A}]$ is expressed as:

$$
\left[\mathrm{A}_{k}\right]=\left[\begin{array}{cccc}
\frac{R_{1}}{L_{1}}\left(-1+\frac{R_{1}}{Z_{0}+\sum_{i=1}^{n} R_{i}}\right) & \frac{R_{1}}{L_{1}}\left(\frac{R_{2}}{Z_{0}+\sum_{i=1}^{n} R_{i}}\right) & \ldots & \frac{R_{1}}{L_{1}}\left(\frac{R_{n}}{Z_{0}+\sum_{i=1}^{n} R_{i}}\right) \\
\frac{R_{2}}{L_{2}}\left(\frac{R_{1}}{Z_{0}+\sum_{i=1}^{n} R_{i}}\right) & \frac{R_{2}}{L_{2}}\left(-1+\frac{R_{2}}{Z_{0}+\sum_{i=1}^{n} R_{i}}\right) & \ldots & \frac{R_{2}}{L_{2}}\left(\frac{R_{n}}{Z_{0}+\sum_{i=1}^{n} R_{i}}\right) \\
\vdots & \ddots & \vdots \\
\frac{R_{n}}{L_{n}}\left(\frac{R_{1}}{Z_{0}+\sum_{i=1}^{n} R_{i}}\right) & \frac{R_{n}}{L_{n}}\left(\frac{R_{2}}{Z_{0}+\sum_{i=1}^{n} R_{i}}\right) & \ldots & \frac{R_{n}}{L_{n}}\left(-1+\frac{R_{n}}{Z_{0}+\sum_{i=1}^{n} R_{i}}\right)
\end{array}\right]
$$

Matrix $\left[\mathrm{B}_{k}\right]$ is also developed as a function of $Z_{0}$ and from the $R$ and $L$ tabulated values of the circuit in Figs. 3 and 6. Matrix $\left[B_{k}\right]$ has dimension $n$ per 2, as in (24).

$$
\left[\mathrm{B}_{k}\right]=\left[\begin{array}{cc}
\frac{R_{1}}{L_{1}}\left(\frac{1}{Z_{0}+\sum_{i=1}^{n} R_{i}}\right) & \frac{R_{1}}{L_{1}}\left(\frac{Z_{0}}{Z_{0}+\sum_{i=1}^{n} R_{i}}\right) \\
\frac{R_{2}}{L_{2}}\left(\frac{1}{Z_{0}+\sum_{i=1}^{n} R_{i}}\right) & \frac{R_{2}}{L_{2}}\left(\frac{Z_{0}}{Z_{0}+\sum_{i=1}^{n} R_{i}}\right) \\
\vdots & \vdots \\
\frac{R_{n}}{L_{n}}\left(\frac{1}{Z_{0}+\sum_{i=1}^{n} R_{i}}\right) & \frac{R_{n}}{L_{n}}\left(\frac{Z_{0}}{Z_{0}+\sum_{i=1}^{n} R_{i}}\right)
\end{array}\right]
$$

Eq. (25) describes the vector [S], which is composed of the voltage source $V_{S}$, that represents the time-variable input voltage $V_{\text {in }}(t)$, and the historical current source $I_{k}(t-\tau)$.

$[S]=\left[\begin{array}{c}V_{\text {in }}(t) \\ I_{k}(t-\tau)\end{array}\right]$

From the state-space formulation in (20), current $i_{k, m}(t)$ and voltage $V_{k}(t)$ can be analytically expressed as follows:

$$
\begin{aligned}
i_{k, m}(t)= & \sum_{q=1}^{n}\left(\frac{R_{q} i_{k q}}{Z_{0}+\sum_{i=1}^{n} R_{i}}\right)+\frac{1}{Z_{0}+\sum_{i=1}^{n} R_{i}} V_{i n} \\
& +\frac{Z}{Z_{0}+\sum_{i=1}^{n} R_{i}} I_{k}(t-\tau) \\
V_{k}(t)= & V_{i n}(t)-i_{k, m}(t) \sum_{i=1}^{n} R_{i}\left(1+i_{k i}\right)
\end{aligned}
$$

The formulation for the node $m$ is similar to the development obtained for the node $k$, since the parameters of the equivalent circuit $Z_{\text {fit }}(\omega)$ are the same for both sides of the equivalent circuit in Fig. 6. Considering the node $m$ as the receiving end of the line, a generic load, represented by an impedance $Z_{\mathrm{L}}$, is included in the state matrix $\left[A_{m}\right]$ as described in (28). 


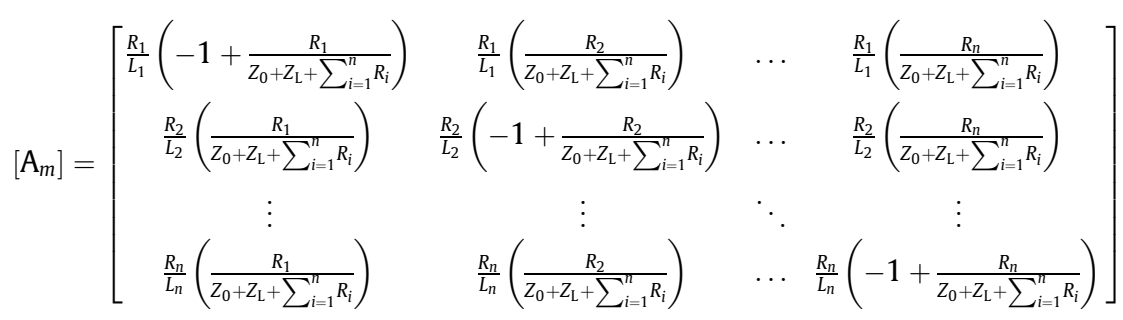

The load $Z_{\mathrm{L}}$ can be modeled as a constant or time-variable impedance, since the proposed line model is developed in the time domain.

The vector with the currents through inductors $L_{1}$ to $L_{n}$ could be renamed as $\left[\mathrm{I}_{m}\right]$; however:

$\left[\mathrm{I}_{m}\right]^{T}=\left[\mathrm{I}_{k}\right]^{T}=\left[\begin{array}{llll}i_{k 1} & i_{k 2} & \ldots & i_{k n}\end{array}\right]$

and

$\left[\dot{\mathrm{I}}_{m}\right]^{T}=\left[\dot{\mathrm{I}}_{k}\right]^{T}=\left[\frac{d i_{k 1}}{d t} \frac{d i_{k 2}}{d t} \ldots \frac{d i_{k n}}{d t}\right]$.

Thus, vector $\left[\mathrm{I}_{m}\right]$ can be expressed only in terms of $i_{k}$.

Terms [B] and [S], in (20), are also modified. Matrix [B] is reformulated as a single vector composed of a single column:

$\left[\mathrm{B}_{m}\right]=\left[\begin{array}{c}\frac{R_{1}}{L_{1}}\left(\frac{Z_{0}}{Z_{0}+Z_{\mathrm{L}}+\sum_{i=1}^{n} R_{i}}\right) \\ \frac{R_{2}}{L_{2}}\left(\frac{Z_{0}}{Z_{0}+Z_{\mathrm{L}}+\sum_{i=1}^{n} R_{i}}\right) \\ \vdots \\ \frac{R_{m}}{L_{m}}\left(\frac{Z_{0}}{Z_{0}+Z_{\mathrm{L}}+\sum_{i=1}^{n} R_{i}}\right)\end{array}\right]$

Vector [S], in (20), is substituted by the past-history current source $I_{m}(t-\tau)$ from the node $m$. Thus, the state equation in (20) can be rewritten from the current source in node $m$ of the equivalent circuit in Fig. 6:

$\left[\dot{\mathrm{I}}_{k}\right]=\left[\mathrm{A}_{m}\right]\left[\mathrm{I}_{k}\right]+\left[\mathrm{B}_{m}\right] I_{m}(t-\tau)$

From the Bergeron model representation by state equations, current $i_{k, m}(t)$ and voltage $V_{m}(t)$ are analytically expressed based on the same development in (26) and (27):

$i_{k, m}(t)=\sum_{q=1}^{n}\left(\frac{R_{q} i_{k q}}{Z_{0}+Z_{\mathrm{L}}+\sum_{i=1}^{n} R_{i}}\right)+\frac{Z_{0}}{Z_{0}+Z_{\mathrm{L}}+\sum_{i=1}^{n} R_{i}} I_{m}(t-\tau)$

$V_{m}(t)=V_{\text {out }}(t)-i_{m, k}(t) \sum_{i=1}^{n} R_{i}\left(1+i_{k i}\right)$

Where the output voltage $V_{\text {out }}(t)$ is expressed as:

$V_{\text {out }}(t)=-i_{m, k}(t) Z_{\mathrm{L}}$

Since the state matrixes are obtained from the fitted parameters ( $R$ and $L$ elements of the equivalent circuit in Fig. 3 ), the first-order system with the state equations can be solved using numerical or analytical integration methods [7].

\section{Cascade representation of the frequency-dependent Bergeron model}

As mentioned before, the cascade approach of the line represents more accurately the distributed characteristic of the line electrical parameters. Furthermore, the frequency response of the model is proportional to the number of equivalent circuits used in cascade [7]. Thus, the cascade representation shows to be a good technique to model long transmission lines for simulation of fast and impulsive electromagnetic transients, which are composed of a wide range of frequencies.

In Section 'Frequency-dependent Bergeron line model', the state matrices were developed for a single frequency-dependent Bergeron circuit. This section shows the matrix formulation for the interconnection of two consecutive elements in cascade with $h$ frequency-dependent Bergeron circuits. From the matrix equations described in Section 'Frequency-dependent Bergeron line model' and based on the state matrices to be developed in this section, the frequency-dependent Bergeron formulation can be extended for a cascade with $h$ equivalent circuits.

Firstly, a cascade with $h$ sections is illustrated in Fig. 7.

Each block in Fig. 7 represents a frequency-dependent Bergeron circuit. The left hand of the first Bergeron circuit represents the sending end of the line whereas the right hand of the last Bergeron circuit represents the receiving end.

Based on the generic frequency-dependent Bergeron circuit in Fig. 6, the connection of circuits $(h-1)$ and $h$ can be formulated. As a first consideration, the $Z_{\text {fit }} / 2$ circuit of the receiving end of $(h-1)$ is exactly the same $R L$ circuit of the sending end of the element $h$. Thus, the equivalent $R L$ circuit obtained from the connection of two consecutive blocks (Fig. 7) is $Z_{\text {fit }}$. Thus, the current $I_{m}$ of the receiving end of the $(h-1)$-th circuit and the current $I_{k}$ from the sending end of the $h$-th circuit are in the same $R L$ circuit. This two current are expressed as $I_{m}^{(h-1)}$ and $I_{k}^{(h)}$, respectively.

The sum of the two $R L$ circuits of the elements $(h-1)$ and $h$ results in a few variations in the state matrix [B] and vector [S], such as expressed in (20) and (32). From the equivalent formation rule based on the Kirchoff's current law in (19), the matrix $\left[B_{m k}\right]$ and vector $\left[S_{m k}\right]$ are expressed in (36) and (37), respectively. On the other hand, matrix $[A]$ remains the same as $\left[A_{k}\right]$.

$$
\begin{aligned}
& {\left[\mathrm{B}_{m k}\right]=\left[\begin{array}{cc}
\frac{R_{1}}{L_{1}}\left(\frac{Z_{0}}{2\left(Z_{0}+\sum_{i=1}^{n} R_{i}\right)}\right) & \frac{R_{1}}{L_{1}}\left(\frac{-Z_{0}}{2\left(Z_{0}+\sum_{i=1}^{n} R_{i}\right)}\right) \\
\frac{R_{2}}{L_{2}}\left(\frac{Z_{0}}{2\left(Z_{0}+\sum_{i=1}^{n} R_{i}\right)}\right) & \frac{R_{2}}{L_{2}}\left(\frac{-Z_{0}}{2\left(Z_{0}+\sum_{i=1}^{n} R_{i}\right)}\right) \\
\vdots & \vdots \\
\frac{R_{n}}{L_{n}}\left(\frac{Z_{0}}{2\left(Z_{0}+\sum_{i=1}^{n} R_{i}\right)}\right) & \frac{R_{n}}{L_{n}}\left(\frac{-Z_{0}}{2\left(Z_{0}+\sum_{i=1}^{n} R_{i}\right)}\right)
\end{array}\right]} \\
& {\left[S_{m k}\right]=\left[\begin{array}{c}
I_{m}^{(h-1)}(t-\tau) \\
I_{k}^{(h)}(t-\tau)
\end{array}\right]}
\end{aligned}
$$

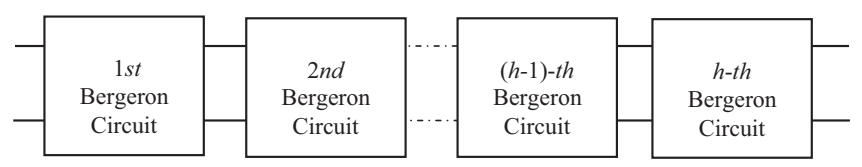

Fig. 7. Cascade of frequency-dependent Bergeron circuits. 
An important characteristic in (37) is the relationship of the past-history current sources $I_{m}^{(h-1)}$ and $I_{k}^{(h)}$. Vector $\left[S_{m k}\right]$ is the main variable to the cascade configuration of the proposed Bergeron model. Considering (36) and (37), the matrix equation of the link between two consecutive Bergeron circuits is expressed in (38).

$\left[\dot{\mathrm{I}}_{k}\right]=\left[\mathrm{A}_{k}\right]\left[\mathrm{I}_{k}\right]+\left[\mathrm{B}_{m k}\right]\left[\mathrm{S}_{m k}\right]$

Analogously to (26), (27) and (33), (34), for a single frequencydependent Bergeron circuit, the current and voltage equations for two connected Bergeron circuits can be analytically developed:

$$
\begin{aligned}
i_{m, k}^{(h-1)}=- & i_{k, m}^{(h)}=\sum_{q=1}^{n}\left(\frac{R_{q} i_{k q}}{Z_{0}+\sum_{i=1}^{n} R_{i}}\right)+\frac{Z_{0}}{2\left(Z_{0}+\sum_{i=1}^{n} R_{i}\right)} \\
& \times\left(I_{m}^{(h-1)}(t-\tau)-I_{m}^{(h)}(t-\tau)\right)
\end{aligned}
$$

Since the past-history current sources $I_{m}^{(h-1)}$ and $I_{k}^{(h)}$ are in the same $R L$ circuit, the current $I_{m, k}^{(h-1)}$, from the receiving end of the circuit $(h-1)$, has the same magnitude of the current $I_{k, m}^{(h)}$ at the receiving end of the circuit $h$.

Voltages at the receiving end of the $(h-1)$-th and the sending end of the $h$-th circuit are expressed respectively as follows:

$V_{m}^{(h-1)}(t)=Z\left[i_{k 0}-I_{m}^{(h-1)}(t-\tau)\right]$

$V_{k}^{(h)}(t)=-Z\left[i_{k 0}+I_{k}^{(h)}(t-\tau)\right]$

Emphasizing that current $i_{k 0}$ is equivalent to $i_{k, m}$ and $i_{m, k}$, which are similar for $(h-1)$-th and $h$-th Bergeron circuits.

From the matrix formulation developed in Section 'Frequencydependent Bergeron line model' and from the formulation presented in this section for two Bergeron circuits interconnected, the detailed voltage and current profile can be obtained along the transmission line considering or not time-variable elements, since the proposed model is totally developed in the time domain.

\section{Validation of the frequency-dependent Bergeron model}

This section aims to evaluate the accuracy of the frequencydependent model based on a $100-\mathrm{km}$ transmission line. In order to validate the proposed model, results are compared to a wellestablished model by distributed parameters using numerical Laplace transforms (NLT). The NLT line model is developed in the frequency domain being accurate and useful to validate other models for simple cases without non-linear and time-variable elements. The NLT model is developed from the distributed parameters of the line in the frequency domain, where timedomain results are obtained applying inverse transforms [2].

Initially, two standard cases are simulated to highlight possible variations between the frequency-dependent Bergeron model (proposed model) and the NLT line model (reference model). The first case consists of a switching impulse applied at the sending end of the transmission line with the receiving end open and in short-circuit. The voltage and current transients are compared based on results obtained from the two models. The second case consists in a transmission line, also with the receiving end open and in short-circuit, submitted to an atmospheric impulse modeled by a voltage double exponential with front-wave time of $1.2 \mu \mathrm{s}$ and tail time of $50 \mu \mathrm{s}$. This input signal is a standard atmospheric impulse proposed by the International Electrotechnical Commission - IEC for high-voltage tests of power devices and systems [13].

Results simulated in this section are obtained using the NLT line model and a cascade with 50 frequency-dependent Bergeron circuits taken into account an impedance $Z_{\text {fit }}(\omega)$ fitted up to $1 \mathrm{MHz}$ (Figs. 4 and 5).
Validation of the proposed model for low frequencies

To validate the proposed model for transients composed of low frequencies, a voltage switching impulse is simulated. The first approach is an unitary step function applied at the sending end of a transmission line with the receiving end open (open-circuit test). It is a conventional test procedure for new line models widely approached in the technical literature on TLM $[1,6-8,10]$.

The physical and geometrical characteristics of the line were previously described in Section 'Fitting the longitudinal impedance' as well as the calculated and fitted resistances and inductances of the line. The parameters were fitted up to $1 \mathrm{MHz}$ using eight $R L$ blocks (Fig. 3), which were calculated using the vector fitting algorithm [12]. Usually, each $R L$ block covers a frequency decade, as described in Figs. 4 and 5.

The transient voltage profiles obtained from the proposed and reference models are shown in Fig. 8.

In Fig. 8a, the result obtained using the proposed model is the dotted curve 1 and the same simulation resulted from the NLT line model (reference model) is the curve 2 . Fig. 8 b shows the detailed profile of both curves in the first wave reflection at the receiving end.

A second test is carried out considering the same input signal at the sending end of the line and the receiving end in short (shortcircuit test). The current transients at the receiving end are described in Fig. 9.

Curve 1 is the current transient simulated by the proposed model and curve 2 is the same transient obtained using the reference model. The transient profile obtained from the proposed model is close to the result simulated using the reference model, since the scale of the vertical axe is in p.u. In the first two or three milliseconds, it is possible to conclude that curves 1 and 2 are almost overlapped.

\section{Validation of the proposed model for high frequencies}

The validation of the proposed model for a wide range of frequencies is carried out by means of an atmospheric impulse applied at the sending end of the line. This input signal is modeled by a voltage source equivalent to a $1.2 / 50 \mu$ s double exponential function. Analogously to tests with a switching impulse in Sectio $\mathrm{n}$ 'Validation of the proposed model for low frequencies', openand short-circuit tests are carried out in this subsection.

First, an atmospheric impulse is applied at the sending end of the line and the voltage transients obtained from both models are presented in Fig. 10.

In Fig. 10, the dotted curve 1 is the voltage transient on the receiving end of the proposed model and curve 2 is the result simulated using the reference model. In Fig. 10a, curves 1 and 2 show to be practically overlapped. In Fig. 10b, a discrete time window is set from 0.4 up to $0.6 \mathrm{~ms}$, highlighting the first wave reflection at the receiving end of the line. A discrete variation in the voltage peak is observed between curves 1 and 2 . However, the difference in the peak magnitude is no more than $2 \%$, which means that the error between proposed and reference models can be neglected.

Results obtained from the short-circuit test, considering an atmospheric impulse at the sending end, are shown in Fig. 11.

The dotted curve 1 and the curve 2 in Fig. 11a are almost overlapped. From a more detailed view, Fig. 11b highlights a discrete variation in the current peak of the curve 1 compared to curve 2 . However, the peak error is no more than $2 \%$.

The validation of the proposed model could be carried out only by an atmospheric impulse, because it covers the entire frequency range considered for fitting $Z_{f i t}(\omega)$. However, to ensure that there are not minor errors in low frequencies, the analysis with a switching impulse shows to be convenient. 


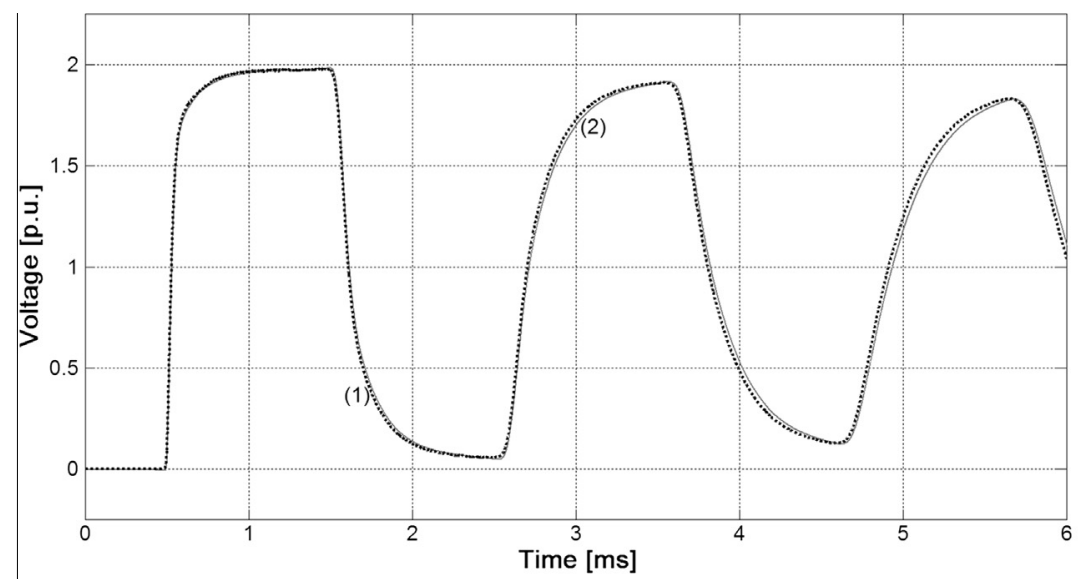

(a)

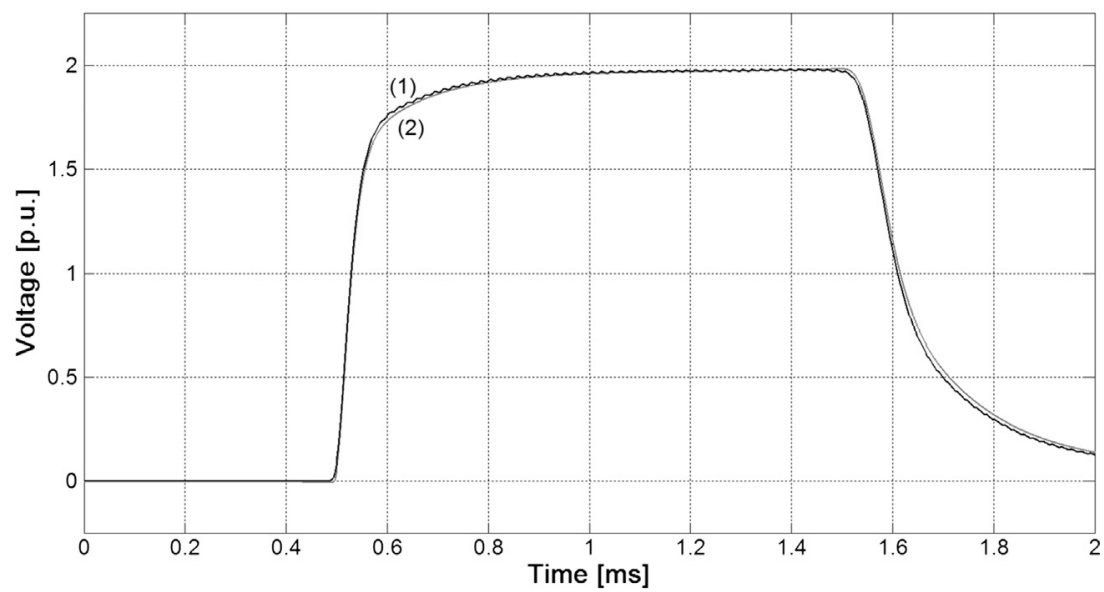

(b)

Fig. 8. Voltage transient at the open receiving end of the line (switching impulse): proposed model (1) and reference model (2).

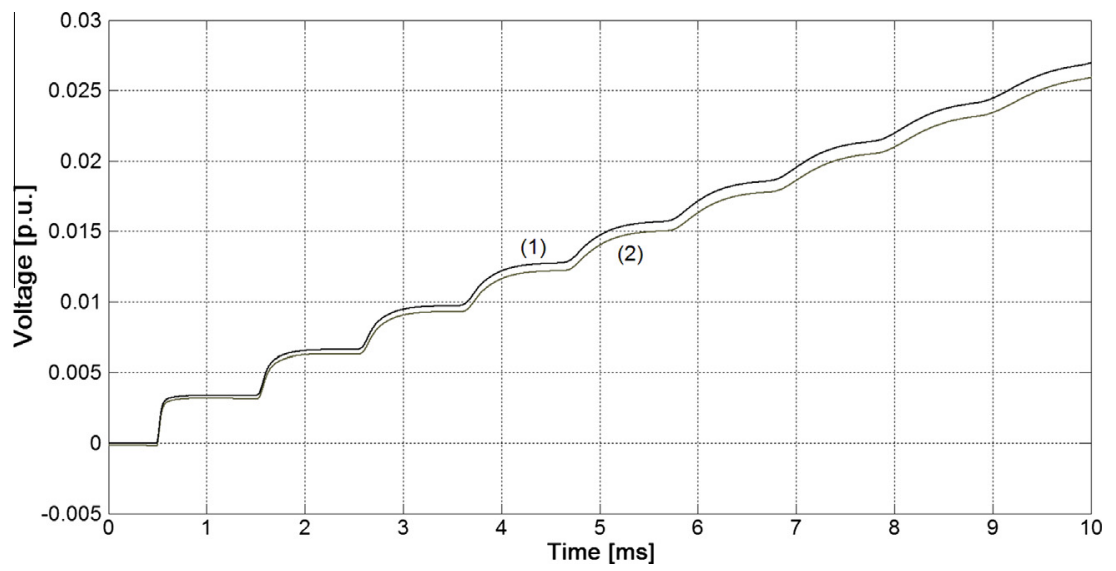

Fig. 9. Current transient at the receiving end of the line in short circuit (switching impulse): proposed model (1) and reference model (2).

Only discrete differences were observed in simulations using the Bergeron's method and the reference model using NLT, which means that the proposed model is valid for simulation of electromagnetic transients composed of low up to very high frequencies (at less up to $1 \mathrm{MHz}$ ).
Simulation of time-variable elements using the frequencydependent Bergeron line model

Since the proposed frequency-dependent model was validated, the next step is an analysis considering time-variable elements in the line modeling and simulations. 


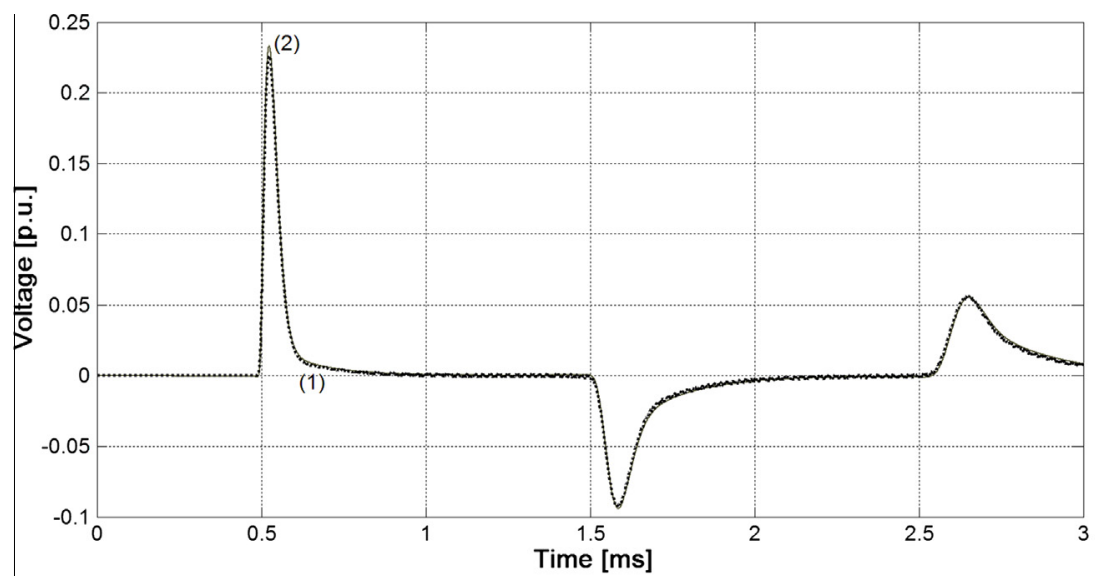

(a)

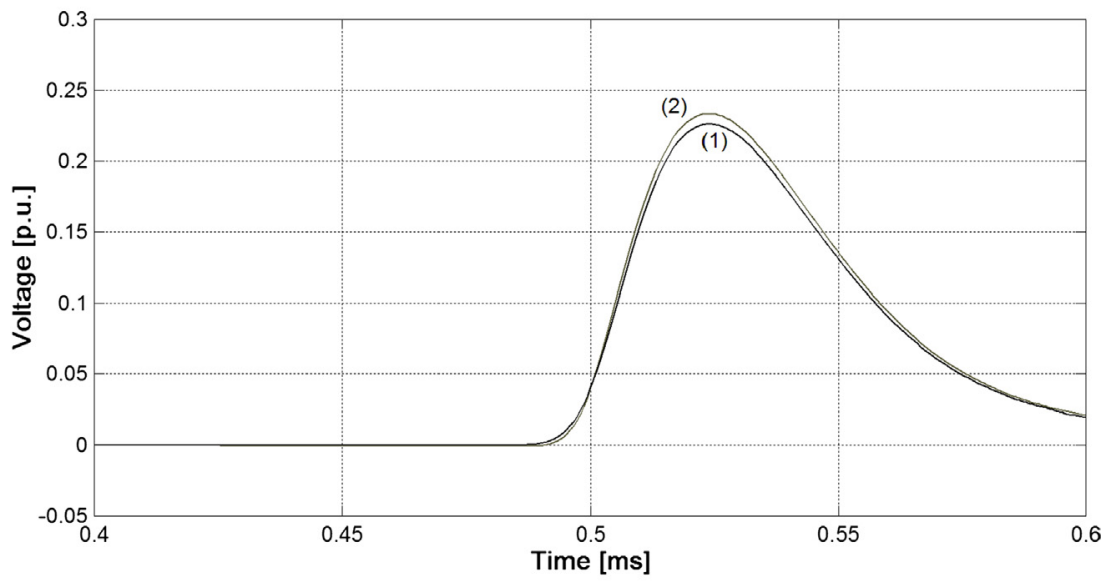

(b)

Fig. 10. Voltage transient at the open receiving end of the line (atmospheric impulse): proposed model (1) and reference model (2).

The possibility of inclusion other power devices in transmission line models is an important characteristic to simulate many operation conditions which power transmission systems are subject. The main issue is that many of these power devices and electromagnetic phenomena are time variable and have a well-known modeling in the time domain. On the other hand, the frequencydomain modeling of these elements is usually a complex procedure or even unknown.

To verify the performance of the proposed model considering time-variable elements, a sinusoidal voltage source at fundamental frequency of $60 \mathrm{~Hz}$ and peak magnitude of 1 p.u. is connected at the sending end of a transmission line with the receiving end connected to a time-variable load with power factor 0.98 . After approximately $16 \mathrm{~ms}$, the load profile changes to a low impedance circuit. Thus, two time-variable events are simulated using the proposed model: a switching followed by load variation.

Fig. 12 shows the voltage profile at the receiving end of the line simulated using the proposed model.

The first transient is observed at the terminal connected to the load, between zero and $5 \mathrm{~ms}$, resulted from the switching impulse applied at the sending end of the line. The second transient, from 15 up to $30 \mathrm{~ms}$, is resulted because the load impedance variation. A low value of the load resistance is set which changes the load characteristics. Fig. 13 shows the current transient occurred due to the switching and load variation.
A careful analysis of the current transient in Fig. 13 shows that the current curve has an inverse signal compared to the voltage transient. This behavior is verified because the current $i_{m, k}$ has a negative signal in the second part of the Bergeron circuit in Fig. 6. The shape and magnitude of the current wave in Fig. 13 is exactly the same, except for the signal.

\section{Conclusions}

The inclusion of the frequency effect in a cascade of Bergeron circuits is the original contribution of this paper. The new Bergeron model was validated based on results simulated using a frequencydomain line model using numerical Laplace transform - NLT. The line model using NLT is developed directly from the line parameters in the frequency domain and the time-domain simulations are carried out applying inverse transforms. The proposed model is validated for frequencies up to $1 \mathrm{MHz}$ considering switching and atmospheric impulses. From the switching impulse was possible to evaluate the proposed model at low frequencies whereas the atmospheric impulse provides a complete scan of frequencies. The line parameters were fitted up to $1 \mathrm{MHz}$.

Voltage and current transients obtained using both line models show to be similar in shape and magnitude, which prove the accuracy of the proposed time-domain model. The cascade of Bergeron 


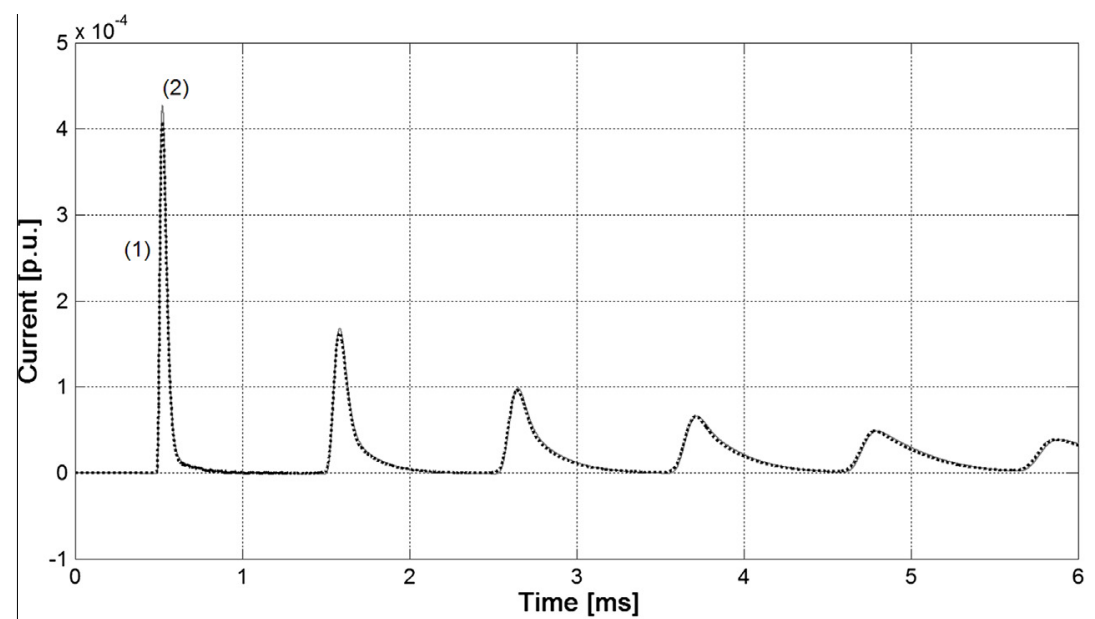

(a)

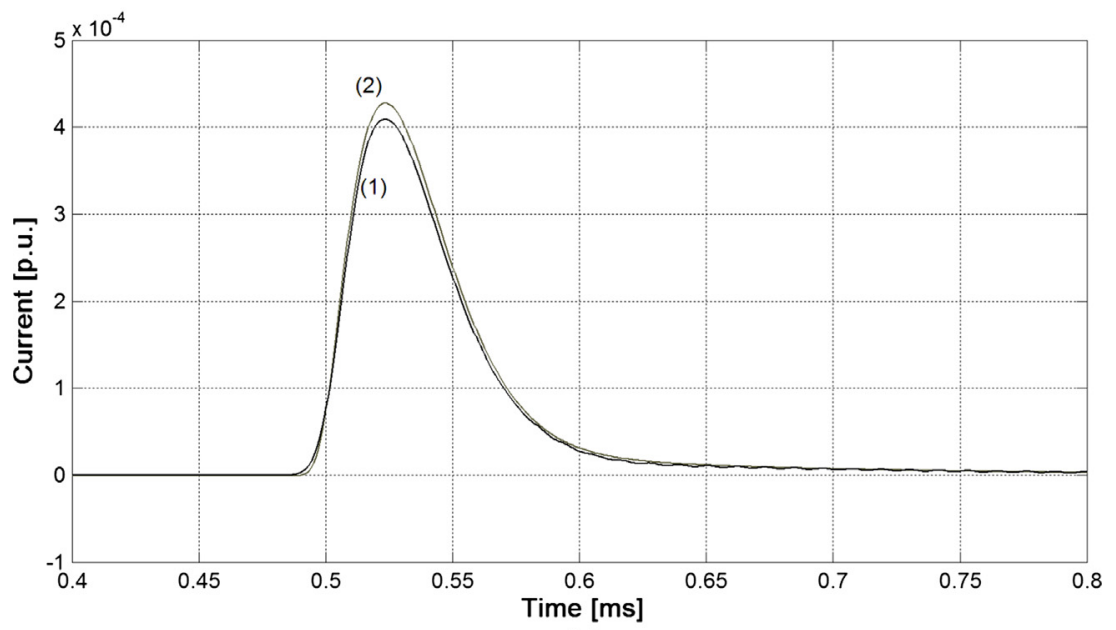

(b)

Fig. 11. Current transient at the receiving end of the line in short circuit (atmospheric impulse): proposed model (1) and reference model (2).

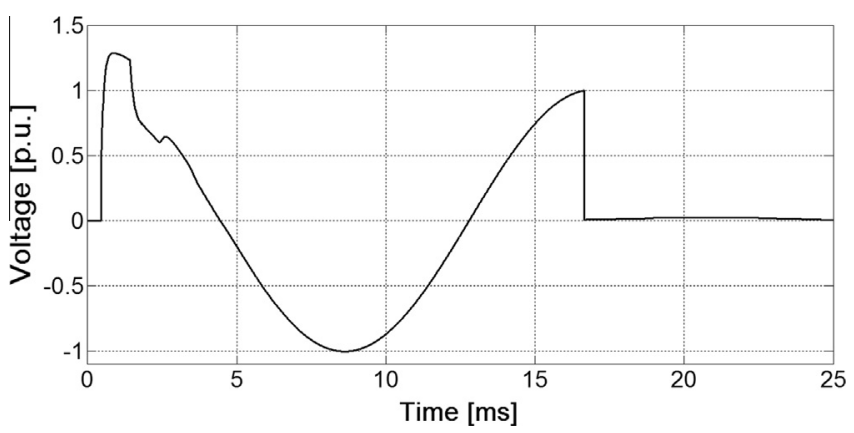

Fig. 12. Voltage transient at the receiving end of the line considering a timevariable load.

circuits presents several advantages compared to the reference model (NLT line model), some of them are listed as follows:

- simplified modeling in the time domain which enables the inclusion of time-variable elements during simulations;

- the cascade representation provides a detailed voltage and current profile along the line as well as the inclusion of non-linear elements at any point through the line length;

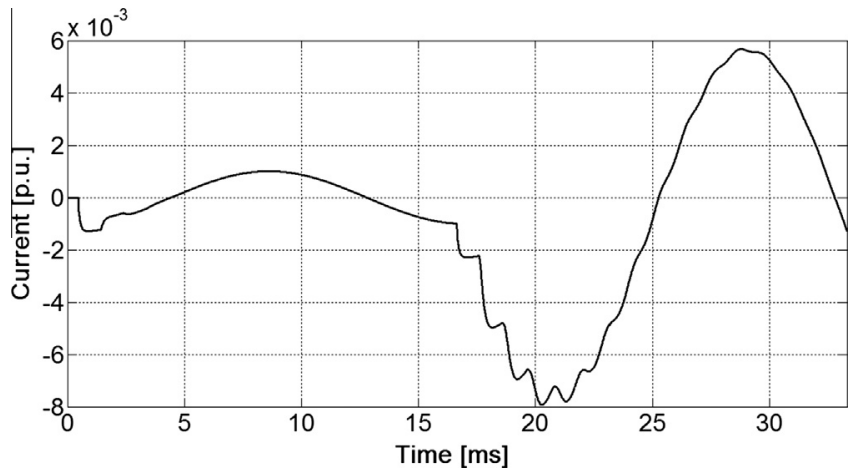

Fig. 13. Current transient at the receiving end of the line considering time-variable load.

- accurate results even for transients composed of a wide range of frequencies;

- inverse transforms and convolutions are not necessary for timedomain simulations.

Besides the referred attributes, the proposed model shows to be accurate and at the same time versatile for inclusion of other power devices in the system modeling. 
As a further development, the proposed model can be extended for multiconductor transmission lines by using modal decoupling techniques. This way, a three-phase line is decoupled into three independent propagation modes that can be modeled as three single-phase lines, using the same frequency-dependent Bergeron model proposed in this paper.

\section{Acknowledgement}

São Paulo Research Foundation - FAPESP (Procs. 14/17051-0 and 15/10204-8).

\section{References}

[1] Mamis MS, Meral ME. State-space modeling and analysis of fault arcs. Electr Power Syst Res 2005;26:46-51.

[2] Di Santo SG, Pereira CEM. Fault location method applied to transmission lines of general configuration. Int J Electr Power Energy Syst 2015;69:287-94.

[3] Santos ML, Di Santo SG. Evaluation of voltage and current profiles and Joule losses for a half-wavelength power transmission line. Int J Electr Power Energy Syst 2015:39-44.
[4] Costa ECM, Kurokawa S. Estimation of transmission line parameters using multiple methods. IET Gener Trans Distrib 2015;9:2617-24.

[5] Gómes P, Uribe FA. The numerical Laplace transform: an accurate technique for analyzing electromagnetic transients on power system devices. Int J Electr Power Energy Syst 2009;31(2-3):116-23.

[6] Mamis MS. Computation of electromagnetic transients on transmission lines with nonlinear components. IEE Proc Gener Trans Distr 2003;150 (2):200-4.

[7] Costa ECM, Kurokawa S, Shinoda AA, Pissolato J. Digital filtering of oscillations intrinsic to transmission line modeling based on lumped parameters. Int J Electr Power Energy Syst 2013;44:908-15.

[8] Costa ECM, Kurokawa S, Prado AJ, Pissolato J. Proposal of an alternative transmission line model for symmetrical and asymmetrical configurations. Int J Electr Power Energy Syst 2011;33:1375-83.

9] Branin HF. Computer methods of network analysis. Proc IEEE 1967;55:1787-801.

[10] Dommel HW. Electromagnetic transient program reference manual (EMTP theory book). Vancouver, Canada: Dept. Electrical Engineering, University of British Columbia; 1989.

[11] Snelson JK. Propagation of travelling waves in transmission lines: frequency dependent parameters. IEEE Trans Power App Syst 1972;PAS-91:85-91.

[12] Gustavsen B, Semlyen A. Combined phase and modal calculation of transmission line transients based on vector fitting. IEEE Trans Power Deliv 1998;13(2):596-604.

[13] IEC 60060-1. High-voltage test techniques - Part 1: General definitions and test requirements. Ed. 3; 2010 . 